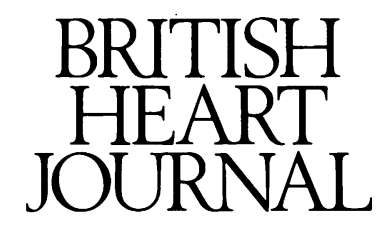

Editorial

\title{
Antioxidant nutrients, atherosclerosis, and coronary heart disease
}

Free radical formation may be causally related to several of the most common diseases, particularly heart disease and cancer. Free radicals can disrupt the function of numerous molecules. They are a chemical species with one or more unpaired electrons. The most harmful free radicals are unpaired oxygen (stable molecules contain paired electrons), hydroperoxides and superoxide anions. Free radicals develop widely in mammalian cells but they are precisely controlled by several antioxidant protective mechanisms. ${ }^{1}$ These include primary enzymatic defences, such as superoxide dismutase and glutathione peroxidase, which depend functionally on the minerals manganese, copper, zinc, and selenium; and stabilising substances such as vitamin $E$ ( $a$-tocopherol), vitamin $C$, $\beta$-carotene, and vitamin $\mathrm{A}$. When the formation of free radicals is not fully prevented or when free radicals are present in excess, they can attack DNA (hence concerns about their relation to cancer), disrupt the function of vital enzymes, and lead to peroxidation of polyunsaturated fats in cell membranes.

An unbalanced excess of free oxygen radicals caused by a lack of antioxidants could increase the risk of coronary heart disease (CHD) in several ways. First, the development of atheroma is partly dependent on incorporation of oxidised cholesterol into monocytes and macrophages within the arterial wall ${ }^{2}$; there are receptors on monocytes and macrophages which do not recognise native low density lipoproteins (LDL) but do recognise modified and oxidised forms of LDL. The susceptibility for oxidation of LDL depends on the stability of the polyunsaturated fatty acids esterified to it, the availability of antioxidants at tissue level, and the density of the LDL molecule. An increase in $a$-tocopherol increases the resistance of LDL to oxidation. ${ }^{3}$ Secondly, platelet adhesion, so important in thrombosis, is more likely when the antioxidant status is low. ${ }^{4}$ Thirdly, the arterial endothelium is protected against damage in primates by dietary $a$-tocopherol. ${ }^{5}$ And, fourthly, unopposed free radical attack within the ischaemic myocardium can impair its function and repair.

Therefore, antioxidant nutrients that protect tissues against attack by free oxygen radicals are potentially important therapeutically. Our food contains many naturally occurring antioxidants. The most powerful are vitamin E ( $a$-tocopherol), vitamin C (ascorbate), and $\beta$ carotene.

Evidence that low intakes of antioxidants are relevant to the development of CHD comes from four sourcesepidemiological data, case-control studies, observational surveys of the effects of supplementation in selected populations, and two controlled trials.

\section{Epidemiology}

The incidence of coronary heart disease, particularly in Europe, is inversely related to the plasma concentration of vitamin $\mathrm{E}$ and, to a lesser extent, to that of vitamin $\mathrm{C}$. A European survey, although not random in sampling and not necessarily representative of the populations under study but based on the known excess of CHD mortality in northern countries, showed a significant inverse correlation $\left(r^{2}=-0.62\right)$ between plasma vitamin E concentrations (lipid standardised) and CHD, and a weaker inverse association $\left(r^{2}=-0 \cdot 11\right)$ for vitamin $C^{6}{ }^{6}$ For both vitamins together the correlation was even stronger $\left(r^{2}=-0.73\right)$. In countries where the incidence of CHD is very high, such as Scotland, Northern Ireland, and Finland plasma concentrations of vitamin $\mathbf{E}$ are significantly lower than in Mediterranean countries where the incidence of CHD is low and consumption of vitamin E, mostly from olive and cereal oils, and citrus fruit containing vitamin $\mathrm{C}$ is high. ${ }^{7}$

In the United Kingdom there are marked differences in vitamin $E$ intake. Social classes III, IV, and V have particularly low intakes of vitamin $E$ and vitamin $C$ because they do not eat many vegetables and fruit. Thus the 1990 Dietary Nutritional Study of British Adults ${ }^{8}$ reports that the $a$-tocopherol/cholesterol ratio was 4.5 in the North of England and 4.7 in the London region $(\mathrm{P}<$ 0.05 ) and 4.8 in social classes I and II compared with 4.4 or less in classes III, IV, and V $(P<0.01)$. It was 4.1 in those who were unemployed, compared with 4.7 in the employed $(P<0.01)$. These differences are most marked in urban communities.

A relatively low intake of antioxidant vitamins, especially in smokers, probably contributes to the higher incidence of CHD in northern England, Scotland, and Glasgow. Cigarette smokers eat fewer cereals and cereal products (the main dietary source of vitamin E) and fruit and vegetables (vitamin C). ${ }^{9}$ The OPCS Study showed that those who smoked more than 20 cigarettes a day had a $a$-tocopherol/cholesterol ratio of $4 \cdot 2$ compared with 4.8 in non-smokers $(P<0.01) .{ }^{8}$ Diet may partly explain the positive relation between smoking and CHD.

The potent antioxidant activity of polyphenols has been studied. These include flavonoids (such as quercetin) which occur naturally in vegetables, fruits, tea, and red wine; quercetin, for example, inhibits the oxidation and cytotoxicity of oxidised LDL in vitro. The Zutphen Elderly Study showed an inverse relation between flavonoid intake and CHD mortality and nonfatal myocardial infarction. ${ }^{10}$ The intakes of tea, onions, and apples, which are all sources of flavonoids, were also inversely correlated with CHD mortality in this study. A 
high consumption of red wine, which has a high content of flavonoids and reduces LDL cholesterol oxidation in vitro, ${ }^{11}$ has been proposed as one of the explanations of the French paradox of low CHD rates in a country with a high consumption of animal fat and excess cigarette smoking. There is no doubt that serum antioxidant activity increases after drinking red wine. ${ }^{12}$

\section{Angina and myocardial infarction}

A case-control study of patients with recent onset angina and controls derived from a high incidence population of 6000 men in Scotland has shown that the risk of developing angina was strongly related to low ratios of plasma vitamin $\mathrm{E} / \mathrm{cholesterol}$ (relative risk adjusted for other risk factors for the lowest quintile $=2 \cdot 2$ ), and, to a lesser extent vitamin $C(R R=1.6)$ and $\beta$-carotene $(R R=$ $1 \cdot 4) .{ }^{13}$ No correlation was found for vitamin A. A similar relation was shown for patients with a first myocardial infarct and no preceding angina. The relation between low vitamin $\mathrm{E}$ and these clinical manifestations of $\mathrm{CHD}$ was independent of cigarette smoking, although the plasma concentrations of all the antioxidant vitamins were lower in smokers. The effect of smoking on vitamin C concentrations was particularly pronounced.

The Scottish Heart Study showed that among men aged 40-59 who had the highest coronary mortality (SMR 136) $17 \%$ ate no green vegetables and $30 \%$ no fruit. ${ }^{14}$ These figures contrast with those who had the lowest coronary mortality (SMR 61), with $6 \%$ eating no greens and $13 \%$ eating no fruit.

\section{Vitamin supplementation}

OBSERVATIONAL STUDIES

There have been several large observational studies collecting data from populations in which the incidence of CHD is under study.

The US Nurses' Health Study of more than 87000 women suggests that, among those who were middle aged, the use of vitamin E supplements is associated with a reduced incidence of CHD. ${ }^{15}$ This study was conducted through postal dietary questionnaires and it also recorded multivitamin intake. The follow up lasted up to eight years. The relative risk of major coronary disease was 0.54 and of cardiovascular mortality was 0.58 for those taking vitamin $\mathrm{E}$ and 0.88 and 0.77 , respectively, for those taking multivitamins. The interpretation is difficult, because only $15 \%$ of all the nurses took vitamin $\mathrm{E}$ and of these, there were fewer current smokers, more who took vigorous exercise, and more taking hormone replacement therapy. This suggests that those taking vitamin E supplements were not representative of the normal population, were more health conscious, and perhaps more healthy.

Another observational study, also conducted in the United States, of nearly 40000 male health professionals aged 40-75 had a similar design based on dietary questionnaires. ${ }^{16}$ The multivariate relative risk was 0.64 in those consuming more than $60 \mathrm{IU}$ of vitamin $\mathrm{E}$ as compared with those taking less than 7.5 IU per day. It was $0.63(\mathrm{P}<0.001)$ for men taking $100 \mathrm{IU}$ or more compared with those not taking any supplements. The apparent protection was evident throughout the 10 years follow up and was most significant in current smokers. The authors conclude, nevertheless, that the association is weak and that it is not possible to rule out confounding (as for the Nurses' Health Study). In this study, there was no association between the incidence of CHD and $\beta$ carotene or vitamin $\mathrm{C}$ intakes.
An observational survey conducted in 10 centres in Europe-the EURAMIC (European Study on Antioxidants, Myocardial Infarction and Cancer of the Breast) study-showed a significant inverse relation between $\beta$-carotene concentrations in adipose tissue and the development of first myocardial infarction in nearly 1500 previously healthy men. ${ }^{17}$ The odds ratio for the lowest quintile of $\beta$-carotene was 2.62 (the multivariate odds ratio was $1.78, P<0.001$ ). There was no relation between adipose $a$-tocopherol and myocardial infarction, although selenium may have had a protective effect when $a$-tocopherol was low. The patients were recruited from coronary care units after their first myocardial infarction; controls for this study were subjects without a history of infarction. Inclusion of $a$-tocopherol as a continuous variable strengthened the multivariate inverse relation between $\beta$-carotene and myocardial infarction. In this study, both $\beta$-carotene and $a$-tocopherol were negatively associated with cigarette smoking.

\section{CONTROLLED TRIALS}

Two controlled trials of antioxidant action have recently been published. A Finnish trial used antioxidant vitamins $^{18}$ and a Swedish trial used the antioxidant drug, probucol. ${ }^{19}$ Neither showed any cardiovascular benefit.

The Finnish study was a randomised double blind primary prevention trial of vitamin $\mathrm{E}$ or $\beta$-carotene or both in more than 29000 male smokers in Finland aged 50-69. The follow up was from five to eight years. The daily dose of vitamin $\mathrm{E}$ was $50 \mathrm{mg}$ and that of $\beta$-carotene $20 \mathrm{mg}$. The primary aim was to determine the effects of these supplements on the incidence of lung cancer (which was not reduced). The trial also recorded coronary mortality. There was no difference in the CHD rate in the group treated with vitamin $\mathrm{E}$ (mortality rate = 71.0 ) and the control group (75.0) but more haemorrhagic strokes in the vitamin E group. There were more coronary deaths in those who took $\beta$-carotene supplements (mortality rate $=77 \cdot 1$ ) than in the control group $(68 \cdot 9)$. The authors comment that an explanation for the lack of effect of these supplements on the incidence of lung cancer and coronary heart disease might be the low concentrations of vitamins used but they were sufficient to lead to a $40 \%$ increase in serum $a$-tocopherol and a much larger increase $(0.17$ to $3.0 \mathrm{mg} / \mathrm{l})$ in $\beta$-carotene concentrations after 3 years.

The Swedish trial studied the effects of a powerful antioxidant, probucol, on atheromatous lesions in the femoral arteries in 274 hypercholesterolaemic patients. There was no greater regression of femoral atherosclerosis, when assessed angiographically, in those treated with probucol than in those given a placebo (added to a cholesterol-lowering diet and cholestyramine), although LDL cholesterol was reduced significantly by probucol. Total high density lipoproteins (HDL) and $\mathrm{HDL}_{2}$ were lowered by probucol and this latter effect may partly explain the negative findings of this study.

\section{Conclusions}

There is a sound scientific basis and rationale for increasing the intakes of vitamin $\mathrm{E}$ and $\mathrm{C}$ to reduce oxidation of LDL and the likelihood of platelet-related thrombosis and to stabilise endothelial function. The evidence suggesting that low levels of both these antioxidant vitamins, particularly vitamin $E$, are associated with a high incidence of CHD is persuasive. Low levels and low dietary intake of these vitamins are especially common in cigarette smokers. 
No firm conclusions should be based on the various observational studies suggesting that antioxidant vitamin supplementation may help to prevent CHD, because some were conducted in unrepresentative populations and may have been confounded by other factors which influenced the incidence of CHD. Also, the amounts of vitamin supplement were different in each study.

Only two controlled trials have been completed and neither has shown that supplements of antioxidant vitamins given to men reduce the incidence of $\mathrm{CHD}$ or atherosclerosis. Further randomised controlled trials of one or more of the antioxidant vitamins are needed. It has been suggested, ${ }^{20}$ and I agree entirely, that general recommendations to the public are not justified until these trials have been completed and show clear benefit. Although large doses (up to $3200 \mathrm{IU} /$ day of vitamin E are safe, ${ }^{21}$ more studies are needed to define the variables influencing absorption of antioxidant vitamins.

The best possible advice is to eat more cereal oils (the main source of vitamin E), olive oil, nuts, fresh citrus fruits (the main source of vitamin $\mathrm{C}$ ), and fresh vegetables and potatoes. Public health advice along these lines needs more emphasis ${ }^{22}$ in populations with high incidence of coronary disease, with especial focus on cigarette smokers. The agricultural and food industries, led by government acceptance of these facts, need to make these foods more readily available and at reduced prices.

MICHAEL F OLIVER

Professor Emeritus,

National Heart and Lung Institute,

London SW3 $6 L Y$

1 Diplock AT. Anti-oxidants and disease prevention. Molec Aspects Med 1994;15:295-376

2 Witztum JL. The oxidation hypothesis of atherosclerosis. Lancet 1994; 344:793-8.

3 Esterbauer H, Striegl G, Puhl H, et al. The role of vitamin E and carotenoids in preventing oxidation of low-density lipoproteins. Ann NY Acad Sci 1989;570:254-67.
4 Salonen JT, Salonen R, Seppanen K, Rinta-Kiika S, et al. Effects of antioxidant supplementation on platelet function: a randomized pairmatched, placebo-controlled, double-blind trial in men with low anti-oxidant status. Am $\mathcal{F}$ Clin Nutr 1991;53:1222-9.

5 Verlangieri AJ, Bush MJ. Effects of d-a-tocopherol supplementation on experimentally induced primate atherosclerosis. $\mathscr{f} \mathrm{Am}$ Coll Nutr 1992;11:131-8.

6 Gey KF, Puska P, Jordan P, Moser UK. Inverse correlation between plasma vitamin $\vec{E}$ and mortality from ischemic heart disease in crossplasma vitamin E and mortality from ischemic heart

7 Riemersma RA, Oliver MF, Elton RA, et al. Plasma antioxidants and coronary heart disease: vitamins $\mathrm{C}$ and $\mathrm{E}$ and selenium. Eur $f$ Clin Nutr coronary heart dise

8 Office of Population and Censuses and Surveys. Dietary nutritional study of British adults London: HMSO, 1990.

9 Fulton M, Thomson M, Elton RA, et al. Cigarette smoking, social class and nutrient intake: relevance to coronary heart disease. Eur $\mathcal{f}$ Clin Nutr 1988;42:797-803.

10. Hertog MGL, Feskens EJM, Hollman PCH, et al. Dietary antioxidan flavonoids and risk of coronary heart disease: the Zutphen Elderly Study. Lancet 1993;342:1007-11.

11 Frankel EN, Kanner J, German GB, et al. Inhibition of oxidation of human low-density lipoprotein by phenolic substances in red wine. Lancet 1993;341:454-7.

12 Maxwell S, Cruickshank A, Thorpe G. Red wine and antioxidant activity in serum. Lancet 1994;344:193-4.

13 Riemersma RA, Wood DA, Macintyre CAA, et al. Risk of angina pectoris and plasma concentrations of vitamins $\mathrm{A}, \mathrm{C}$, and $\mathrm{E}$ and carotene. Lancet and plasma conce

14 Bolton-Smith C, Smith WCS, Woodward M, Tunstall-Pedoe H. Nutrient intakes of different social-class groups: results from the Scottish Heart Health Study (SHHS). Br $\mathcal{F}$ Nutr 1991;65:321-35.

15 Stampfer MJ, Hennekens CH, Manson JE, et al. Vitamin E consumption and the risk of coronary heart disease in women. N Engl $\mathcal{F}$ Med 1993; 328:1444-9.

16 Rimm EB, Stampfer MJ, Ascherio A, et al. Vitamin E consumption and the risk of coronary heart disease in men. $N$ Engl $f$ Med 1993;328: $1450-6$.

17 Kardinaal AFM, Kok FJ, Gomez-Aracena J, et al. Antioxidants in adipose tissue and risk of myocardial infarction: the EURAMIC Study. Lancet 1993;342:1379-84.

18 The Alpha-Tocopherol, Beta Carotene Cancer Prevention Study Group. The effect of vitamin $E$ and beta carotene on the incidence of lung The effect of vitamin E and beta carotene on the incidence of lung cancer and

19 Walldius G, Erikson U, Olsson AG, et al. The effect of probucol on femoral atherosclerosis: the probucol Quantitative Regression Swedish

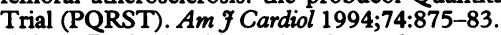

20 Steinberg $\mathrm{D}$. Anti-oxidant vitamins and coronary heart disease. $N$ Engl $\mathcal{F}$ Med 1993;328:1487-9.

21 Bendich A, Machlin LJ. Safety of oral intake of vitamin E. Am f Clin Nut 1988;48:612-9.

22 Report of the Cardiovascular Review Group. Nutritional aspects of cardiovascular disease. COMA. London: HMSO, 1994. 